\title{
ROLL-TO-ROLL CLEANING AND ACTIVATION OF ULTRA-THIN FLEXIBLE GLASS USING ATMOSPHERIC-PRESSURE PLASMA IN AMBIENT AIR
}

\author{
Slavomír SIHELNÍK, Richard KRUMPOLEC, Monika STUPAVSKÁ, Jianyu FENG, Eva KOSOVÁ, \\ Jakub KELAR, Mirko ČERNÁK, Dušan KOVÁČIK \\ Masaryk University, Faculty of Science, Department of Physical Electronics, CEPLANT, Brno, \\ Czech Republic, EU, sihelnik@mail.muni.cz
}

https://doi.org/10.37904/nanocon.2021.4344

\begin{abstract}
Flexible electronics are nowadays manufactured on substrates made particularly of plastics and paper. In demand for better optical and chemical properties, glass is also used in its ultra-thin flexible form (UTFG). Digital ink-jet printing is an attractive method for deposition of conductive precursors on flexible substrates, mainly due to its ability to be incorporated into roll-to-roll systems for massive production. Plasma cleaning and activation is proposed here as a dry, efficient, and controllable method for pre-treatment of glass substrate before deposition of conductive inks. Tested plasma sources were chosen with respect to the specific thermomechanical properties of UTFG and requirements of large area printing on flexible substrates. Both diffuse coplanar surface barrier discharge (DCSBD) and industrial corona achieved a significant wetting improvement of UTFG, which results from modification of properties governed by surface nanolayer. Water contact angle (WCA) measurement and X-ray photoelectron spectroscopy revealed strong activation of UTFG surface due to incorporation of oxygen-based polar groups. The ageing of plasma-treated glass, monitored for one week, showed better stability of the DCSBD plasma-treated UTFG surface. We observed the WCA recovery from $7 \%$ to $38 \%$ in respect to the initial value measured on the untreated UTFG $\left(68^{\circ}\right)$. The impact of plasma on the morphology of the glass surface was analysed using atomic force microscopy.
\end{abstract}

Keywords: Flexible glass, roll-to-roll, cleaning, plasma activation, DCSBD, industrial corona

\section{INTRODUCTION}

Surface modification of glass is a widely used approach of tailoring its properties when interference with its bulk is not desirable or necessary. Tools for glass surface modifications could consist of any state of matter; therefore even plasma treatment has been used over decades, mainly in laboratory environments. Application of plasma treatment could be especially advisable in processes when desired properties are governed by a surface nano-layer [1].

The contemporary flexible electronics are usually made on plastic substrates using coating and printing methods. Paper and flexible glass are other substrate materials researched and applied in this sphere, connected to fields like photovoltaics, biophysics and optoelectronics [2]. Ultra-thin flexible glass (UTFG) is a novel material, and so far, only a few studies reported the application of plasma treatment to its surface. UTFG's potential and its advantageous properties were shown in the studies of indoor photovoltaics, wireless antennas and organic light-emitting diodes (OLED) [3]. In microelectronics and printed flexible electronics manufactured on UTFG, the bonding of thin structures deposited using printed conductive inks is extremely important, and it can be improved by cleaning and chemical activation of the glass surface before deposition.

The exposure of glass surface to ambient air conditions, which causes adsorbing of air pollutants to it, plays an essential role on the scale of molecular size. The naturally adsorbed layer consisting of water and organic 
contamination with a thickness of several nanometers could hinder processes based on adding material to the glass surface. Exposure of glass surface to active species included in plasma seems to be a potential method for fusion of cleaning and activation into one processing step, which can be realized immediately before the deposition of coating, thin film or layered structure. Plasma cleaning of the glass surface is provided through free electrons with energy high enough to break hydrogen bonds of adhered layer contaminating surface [4]. Active species produced by plasma interaction with surrounding gas can be incorporated onto the plasmatreated surface during the exposure. Activation of the UTFG surface by promoting functional groups generated in plasma could facilitate the coupling of precursor molecules in ink-jet printing processes.

The choice of a useful plasma system that can be applied on materials like UTFG is limited by its thermal and mechanical sensitivity. Different non-thermal plasma sources need to be tested and compared to obtain desirable modifications that could be suitable for improving concrete applications on UTFG. The impact of parameters like discharge regime, exposure time or distance are tested to achieve better efficiency, uniformity and stability of the effect, which can be analyzed through wetting measurements and XPS analyses of chemical composition. The influence of plasma interaction to morphology of treated glass surface is analyzed with AFM measurements of roughness.

\section{EXPERIMENT}

UTFG AF32 sheets with a thickness of $30 \mu \mathrm{m}$ and a size of $101.6 \times 101.6 \mathrm{~mm}$ were purchased from Schott AG. AF32 samples of this original waffer size were used only in the experiments in planar configuration. Other UTFG samples were been cut to form of bands $12 \times 80 \mathrm{~mm}$ in the order to prevent conveying errors on R2R systems and thus improve the achieved effect. A part of experiments focused on impact of plasma to surface morphology was realised on common float soda-lime glass (SLG) from Polartherm-Flachglas GmbH with $2.8 \mathrm{~mm}$ thickness and a size of $20 \times 80 \mathrm{~mm}$. All of the experiments were carried on the side of glass without tin content. UTFG samples were only blown over to eliminate the presence of residuals from laser cutting. Samples of SLG were contamined by wet cutting process, so they were precleaned in ultrasonic cleaner with acetone, isopropyl alcohol and deinoised (DI) water.

Table 1 Setup of applied devices using plasma treatment in ambient air at atmospheric pressure

\begin{tabular}{|c|c|c|c|c|c|}
\hline Device & Geometry & $\begin{array}{c}\text { Dimensions } \\
(\mathbf{m m})\end{array}$ & $\begin{array}{c}\text { Power } \\
(\mathbf{W})\end{array}$ & $\begin{array}{c}\text { Areal power density } \\
\left(\mathbf{W} / \mathbf{c m}^{2}\right)\end{array}$ & $\begin{array}{c}\text { Volume power density } \\
\left(\mathbf{W} / \mathbf{c m}^{3}\right)\end{array}$ \\
\hline DCSBD "Air-pillow" & planar & $80 \times 195$ & 400 & 2.57 & 85.47 \\
\hline Roll-To-Roll DCSBD & curved & $80 \times 195$ & 400 & 2.57 & 85.47 \\
\hline Roll-To-Roll VDBD & curved & $75 \times 210$ & 400 & 2.54 & 25.40 \\
\hline
\end{tabular}

Two basic configurations of dielectric barrier discharge for generating atmospheric pressure plasma in air were been compared. Variations of the used devices employing diffuse coplanar surface barrier discharge (DCSBD) and volume dielectric barrier discharge (VDBD) units and their parameters are summarised in Table 1. Devices using DCSBD plasma units provided by ROPLASS, s. r. o. were tesed in two different geometries, indeed both are respecting demand of contactless treatment on a large area. Experiments realised on small samples with planar DCSBD unit were carried with holder with spacers ensuring distance $0.3 \mathrm{~mm}$ between samples and ceramic that is advisable for effective treatment. DCSBD "Air-pillow" is equipped with pneumatic bearings that enables contactless leading of discharge unit over smooth planes of unlimitted size [1]. Closed-chamber RollTo-Roll DCSBD reactor (TYPE III) employs concavely curved discharge units and is designed for treatment of flexible materials using various working gas [5]. Compared commercial device manufactured by Ahlbrandt System $\mathrm{GmbH}$ is using so-called industrial corona generating VDBD plasma of comparable areal power density as DCSBD [6]. 
The static WCA measurement using $1 \mu \mathrm{l}$ of $\mathrm{DI}$ water was realised with See System by Advex Instruments, s. r. o. The XPS analysis was done with ESCALAB $250 \mathrm{Xi}$ analytic platform by Thermo Fisher Scientific, using Al Ka source and Rowland circle monochromator. Spectra calibration, processing and fitting routines were accomplished in Avantage software. AFM measurements were carried on Ntegra Prima by NTMDT in semi-contact mode using silicone tips HA_NC with curvature radius under $10 \mathrm{~nm}$. Surface roughness was evaluated from 5 measurements on $5 \times 5 \mu \mathrm{m}$ areas that were scanned with $1 \mathrm{~Hz}$ frequency.

\section{RESULTS AND DISCUSSION}

The comparision of water contact angles (WCA) measured repeatedly during one week on UTFG treated 1 and $3 \mathrm{~s}$ with tested plasma sources is presented in Figure 1. The graph illustrates ageing study of samples stored in ambient atmosphere, which was particulary carried out on the first day of experiment-after 0,2 , and 8 hours. The rest of WCAs were measured 1,3 , and 7 days later. The WCA $(68 \pm 2)^{\circ}$ is the initial value of untreated $30 \mu \mathrm{m}$ thick AF32 obtained independently on the analysed side. The most significant wettability improvement of UTFG surface immediately after treatments was achieved even after the shortest applied exposure time $1 \mathrm{~s}$ using both roll-to-roll plasma systems. The lowest WCA drop $(4.6 \pm 0.7)^{\circ}$ acquired after $1 \mathrm{~s}$ exposure with the industrial corona was matched with DCSBD "Air-pillow" when $3 \mathrm{~s}$ exposure was applied. More significant and uniform effect on DCSBD plasma treated UTFG surface was observed with $1 \mathrm{~s}$ exposure using curved unit, when the lowest achieved error affected the measured values resulting in $(5.0 \pm 0.5)^{\circ}$. The treatment time of $3 \mathrm{~s}$ with R2R DCSBD reactor proved less effective, which was caused by charging of the UTFG sample from plasma and consequent impact on its leading under the curved DCSBD ceramic, which was electricaly charged, too.

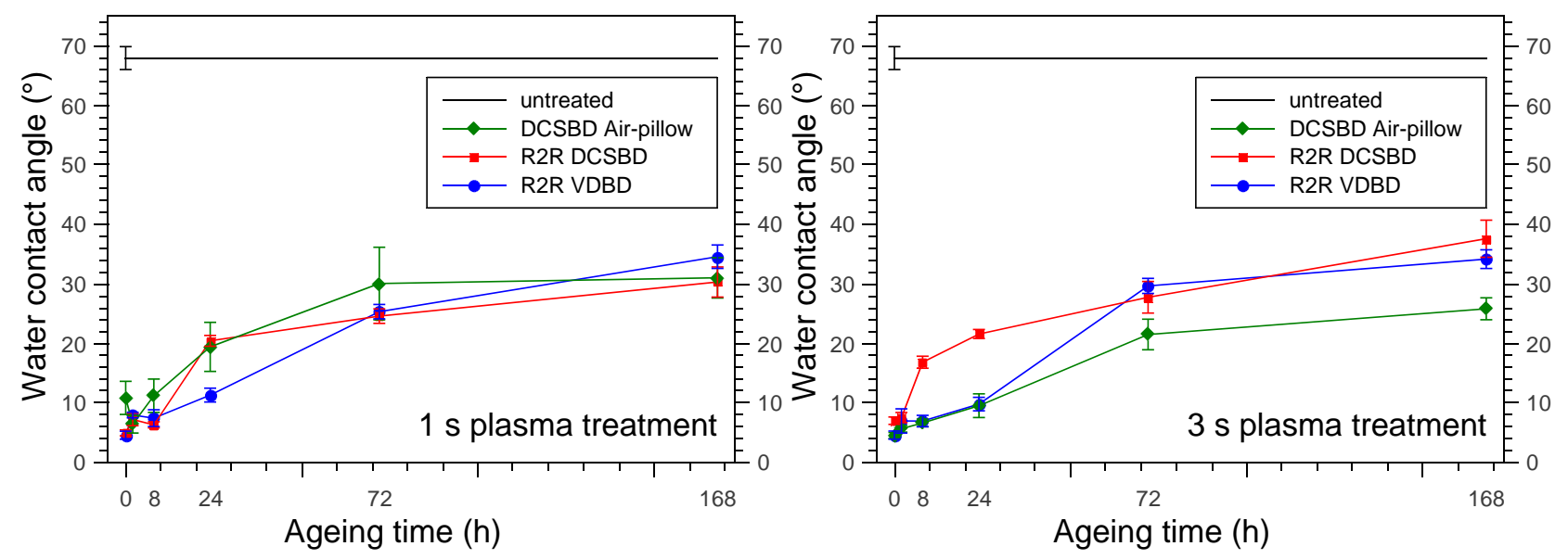

Figure 1 Wettability of ultra-thin flexible glass (UTFG) surface treated with plasma

Hydrophilic effect achieved with roll-to-roll systems was relatively stable in the first 8 hours after exposures of $1 \mathrm{~s}$. The results of experiments applying $3 \mathrm{~s}$ treatment time followed similar tendency, with an exception of values from the R2R DCSBD. Faster recovery of WCAs measured on UTFG treated $3 \mathrm{~s}$ with R2R DCSBD corresponds to the lowering of its efficiency by prolonging treatment time, which was reported immediately after treatment. Stability in the first 8 hours for shorter treatment time is a satisfying result taking into account the duration of one work shift in the industry. Longer storage of plasma-treated UTFG demonstrated gradually recovering tendency, which was more significant in the first 3 days. Afterwards, in the range of 7 days, WCAs have reached a value of only $50 \%$ of the untreated UTFG value for all configurations.

The present study using roll-to-roll plasma treatement with focus on improved conveying of UTFG samples resulted in optimalization of treatment time with curved DCSBD to $1 \mathrm{~s}$, which is closer to industrial requirements, compared to $5 \mathrm{~s}$ from our preliminary study aimed at contactless large area treatment [1]. The 
reported saturation of WCAs measured after 3 days of ageing is in good accordance with the studies using DCSBD on common float glass with exposure times $3 \mathrm{~s}$ [7]. However, the results presented here vary in the initial value of untreated surface, which is much higher, hence changes and stability are relatively more markable on UTFG. Interesting is that minimum and saturation levels are similar independently on the used glass substrate and its initial WCA value. The essence of wettability change of glass surface induced by plasma modification seems to be independent from its initial surface morphology or its eventual alteration by interaction with plasma.

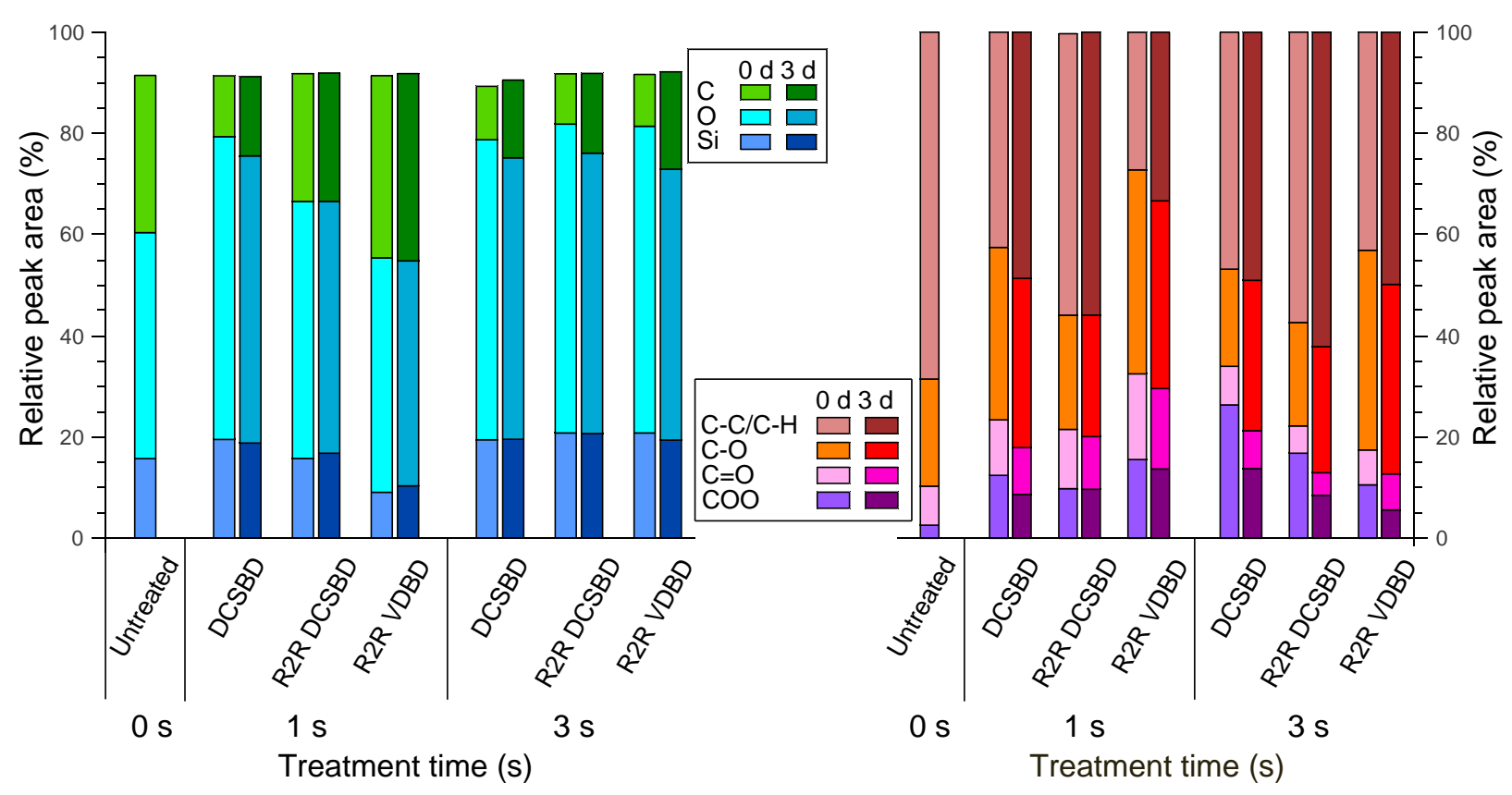

Figure 2 XPS analysis of a) elemental composition and b) carbon bonds partition on UTFG treated with plasma carried before and after 3 days of storage on ambient atmosphere.

The XPS analysis of UTFG treated 1 and $3 \mathrm{~s}$ with all of the tested plasma systems revealed a dominant presence of carbon, oxygen and silicon ( $\mathrm{C} 1 \mathrm{~s}, \mathrm{O} 1 \mathrm{~s}$ and $\mathrm{Si} 2 \mathrm{p})$ in the survey spectra. Atomic concentrations of $\mathrm{C}, \mathrm{O}$ and $\mathrm{Si}$ are presented in Figure 2, their values on untreated sample were $31 \%, 45 \%$ and $16 \%$, respectively. Carbon could be present in organic pollution which is contained in the water film adsorbed to UTFG surface from air humidity. Another possible source of carbon on the plasma-treated UTFG surface are carbonyl and carboxyl functional groups incorporated into it. All the applied plasma treatments induced reduction of atomic carbon concentration, with an exception of $1 \mathrm{~s}$ treatment with VDBD. Storage of 3 days induced alterations of relative carbon content only in the case of longer exposure times $3 \mathrm{~s}$, when it increased from $10 \%$ to $15 \%$ for both DCSBDs and to $19 \%$ for VDBD. The presence of oxygen is related to bulk material, water film and also to the occurrence of chemically active functional groups. After all of the tested plasma treatments, oxygen content increased, while the most significant growth to $61 \%$ was achieved by $3 \mathrm{~s}$ treatment with R2R DCSBD reactor. After 3 days of storage, oxygen concentration is reduced, which was more pronounced for the treatments of $3 \mathrm{~s}$. Silicon concentration represents the bulk material, and its concentration depends on its presence in the analyzable depth profile $(\sim 3 \mathrm{~nm})$. In the carried out experiments, the Si content did not vary so significantly as it for $\mathrm{C}$ and $\mathrm{O}$, which can be explained by their rearrangement on the surface without a markable change in thickness of the surface nano-layer. The achieved results confirmed the expected activation by the rise of oxygen-based groups present after air plasma treatment of even a short duration for both tested plasma sources. However, $1 \mathrm{~s}$ treatment time with VDBD was not enough to decrease atomic carbon relative content, hence its cleaning effect was not achieved, contrarily to the DCSBD plasmas. 
High-resolution spectra of $\mathrm{C} 1 \mathrm{~s}$ peak revealed the principle behind the rearrangement of carbon and oxygen induced by plasma interaction with UTFG surface. Breaking of $\mathrm{C}-\mathrm{C}$ and $\mathrm{C}-\mathrm{H}$ bonds is related to an oxidation process resulting in the bonding of highly reactive oxygen to the surface, which was confirmed by a significant increase of relative peak areas of $\mathrm{C}-\mathrm{O}, \mathrm{C}=\mathrm{O}$ and $\mathrm{O}-\mathrm{C}=\mathrm{O}$ bonds. The acquired data showed an increase of oxygen-based carbon bonds, which was the most significant after $1 \mathrm{~s}$ treatment with VDBD plasma. The fact that atomic carbon content on the UTFG surface increases only after $1 \mathrm{~s}$ VDBD, together with a simultaneous increase of atomic oxygen content implies the assumption that for all other tested conditions the rest of oxygen was bonded directly to the silicone-based structure of UTFGs bulk. Direct oxidation of the silicone structure cleaned from the residuals from organic pollution seems to be more suitable for the improvement of uniformity and stability in time, which is in accordance with the results of WCAs after treatment with R2R VDBD reactor and its uncertainties measured immediately. After 3 days of storage on ambient air, the highest alterations in the $\mathrm{C} 1 \mathrm{~s}$ bonds concentration were shown in $\mathrm{C}-\mathrm{C}$ and $\mathrm{C}-\mathrm{H}$ bonds that increased and $\mathrm{O}-\mathrm{C}=\mathrm{O}$ bonds that decreased approximately by the same relative area. $\mathrm{C}-\mathrm{O}$ and $\mathrm{C}=\mathrm{O}$ bonds changed only slightly, which is in accordance with the higher reactivity of carboxyl groups that were dissolving faster in humid air. Recombination of oxygen-based polar groups in air humidity during storage is in accordance with the decrease of relative peak areas of oxygen-containing bonds and hindering of wettability, which supports the interpretation that the impact of adsorbed water is more pronounced on the sample with a highly oxidised surface. This phenomenon limits the applicability of plasma surface modification, while prolonging exposure does not provide any additional hydrophilic improvement or better stability of the chemical composition of the UTFG surface. The present results of experiments carried out on the R2R DCSBD reactor showed the best match in cleaning rate and activation efficiency for reaching the most desirable effect using treatment with duration of $1 \mathrm{~s}$. Better uniformity and stability of the effect achieved with DCSBD plasmas could be explained with higher local concentrations of active species generated in its thin active plasma region compared to $1 \mathrm{~mm}$ wide gap of VDBD plasma.

Table 2 RMS roughness and arithmetical mean roughness of glass surface treated with DCSBD plasma

\begin{tabular}{|c|c|c|c|c|}
\hline 3-LIQUID CLEANED & $\mathbf{S q}(\mathbf{n m})$ & $\mathbf{u}(\mathbf{S q}) \mathbf{( n m})$ & $\mathbf{S a}(\mathbf{n m})$ & $\mathbf{u}(\mathbf{S a}) \mathbf{( n m})$ \\
\hline Untreated sample & 1.4 & 0.4 & 0.5 & 0.1 \\
\hline DCSBD 3 s & 1.5 & 0.2 & 1.1 & 0.2 \\
\hline DCSBD 10 s & 1.7 & 1.3 & 0.7 & 0.5 \\
\hline
\end{tabular}

Additionally, the DCSBD as the optimal plasma source has been tested for its plasma impact to the morphology of glass surface. Results of AFM measurement on the chemically precleaned samples of common soda-lime glass are summarized in Table 2. Evaluation of root mean square roughness (Sq) and arithmetical mean roughness $(\mathrm{Sa})$ showed significant growth of uncertainties of values obtained in measurements carried out on the sample treated with the longest tested time of $10 \mathrm{~s}$. There was no significant change in Sq of the sample treated for $3 \mathrm{~s}$ with DCSBD, although the resulting Sa values doubled, the uncertainties are significantly lower in comparison to the values measured after $10 \mathrm{~s}$ treatment. The reason of higher uncertainties reported on the samples treated with longer exposure time lies in deterioration of glass surface. The resulting Sq values measured on samples treated with shorter exposure time approved DCSBD plasma as a gentle and uniform instrument for fast surface modification of glass, which seems to be harmless to the bulk glass structure. The achieved results are in accordance with the results reported in former studies, when using DCSBD plasma at shorter exposure times than $10 \mathrm{~s}$ did not cause any significant deterioration of the glass surface [7].

\section{CONCLUSION}

The tested plasma sources achieved a significant wetting improvement of $30 \mu \mathrm{m}$ thick UTFG using optimal treatment time $1 \mathrm{~s}$ with the curved DCSBD unit. WCA and XPS revealed fast pollution removal and strong activation of UTFG surface due to incorporation of oxygen-based polar groups. The ageing of plasma-treated 
UTFG, monitored for one week, showed the best stability of the effect on the DCSBD plasma-treated UTFG surface. Plasma seems to be an efficient and controllable cleaning method that respects the specific thermomechanical properties of UTFG and the requirements of contactless large area printing on fragile flexible substrates processed on roll-to-roll devices.

\section{ACKNOWLEDGEMENTS}

This research has been supported by the projects LM2018097 funded by the Ministry of Education, Youth and Sports (CEPLANT centre) and project FV40114 funded by the Ministry of Industry and Trade (program TRIO).

\section{REFERENCES}

[1] SIHELNÍK, S., KRUMPOLEC, R., STUPAVSKÁ, M., FENG, J., KOSOVÁ, E., KELAR, J., ZEMÁNEK, M., KOVÁČIK, D., ČERNÁK, M. (2020). Large area cleaning and activation of flexible ultra-thin glass by non-thermal atmospheric-pressure plasma. In: Nanocon 2020 Conference Proeedings. [online]. Brno: Tanger, 2020, pp. 305310. Available from: https://doi.org/10.37904/nanocon.2020.3717.

[2] PARK, S., KIM, H., KIM, J. H., \& YEO, W. H. Advanced nanomaterials, printing processes, and applications for flexible hybrid electronics. Materials. [online]. 2020, vol. 13., iss. 16, pp. 1-34. Available from:

https://doi.org/10.3390/MA13163587.

[3] CASTRO-HERMOSA, S., LUCARELLI, G., TOP, M., FAHLAND, M., FAHLTEICH, J., BROWN, T.M. Perovskite Photovoltaics on Roll-To-Roll Coated Ultra-thin Glass as Flexible High-Efficiency Indoor Power Generators. Cell Reports Physical Science. [online]. 2020, vol. 1, iss. 5, pp. 100045 (17). Available from:

https://doi.org/10.1016/j.xcrp.2020.100045.

[4] DEY, T., NAUGHTON, D. Cleaning and anti-reflective (AR) hydrophobic coating of glass surface: a review from materials science perspective. Journal of Sol-Gel Science and Technology. [online]. 2016, vol. 77, iss. 1, pp. 127. Available from: https://doi.org/10.1007/s10971-015-3879-X.

[5] R\&D centre for plasma and nanotechnology surface modifications. DCSBD plasma for the large area roll-to-roll applications (Hydrogen plasma). [online]. 2021 [viewed: 2021-11-01]. Available from: Available from: https://ceplant.cz/equipment-and-services/low-cost-plasma.

[6] Ahlbrandt System GmbH. Corona Pure. [online]. 2021 [viewed: 2021-12-02]. Available from: Available from: https://www.ahlbrandt.com/corona-pure.

[7] BUČEK, Andrej, HOMOLA, Tomáš, ARANYOSIOVÁ, Monika, VELIČ, Dušan, PLECENIK, Tomáš, HAVEL, Josef, ST̃AHEL, Pavel, and ZAHORANOVÁ, Anna. Atmospheric pressure non-equilibrium plasma treatment of glass surface. Chemické Listy. 2008, vol. 102, pp. 1459-1462. 